\title{
Ludwig Winder in der Deutschen Zeitung Bohemia. Prolegomena zu einem tschechoslowakischen
}

\section{Journalisten}

\author{
Ludwig Winder in Deutsche Zeitung Bohemia. \\ Prolegomena to a Czechoslovak Journalist
}

Jan Budňák

\begin{abstract}
Two aims are pursued in this paper. On the one hand, a connecting line between the novels and the essay work of Ludwig Winder should be shown here by way of example, based on the journalistic treatment of 'people of will and power' who are also central to Winder's novels. On the other hand, based on selected journalistic articles in the Deutsche Zeitung Bohemia (DZB), Winder is to be anchored in the cultural and political milieu of the first Czechoslovak Republic. Both goals should arrive to one conclusion: For Ludwig Winder, many more cultural-political contexts are relevant, than just the few in which his writing to date, primarily in Jewish and German literature in Prague, has been put. The context the relevance of which for Winder is outlined in this article is the one that unfolds from the position of a journalist from Czechoslovakia who writes in German (taking into account, however, the culture, politics and literature in Czech language too).
\end{abstract}

\section{Keywords}

Ludwig Winder; essays; German newspapers in Czechoslovakia; pacifism; area studies; German literature from Prague and Bohemia 
Im vorliegenden Beitrag wird zwei Zielen nachgegangen. Zum einen soll hier eine Verbindungslinie zwischen dem Romanwerk und dem journalistischen Werk Ludwig Winders exemplarisch aufzuzeigen sein, und zwar anhand von journalistischer Behandlung von ,Willens-' und/oder ,Machtmenschen', die auch für Winders Romanwerk zentral sind (Wolf Wolf in der Jüdischen Orgel, die Titelfigur in Kasai, Dupic in Die nachgeholten Freuden, Franz Ferdinand d'Este in Thronfolger und viele andere). Zum anderen soll anhand von ausgewählten journalistischen Beiträgen Winders in der Deutschen Zeitung Bohemia (DZB) seine profunde Verankerung im kulturellen und politischen Milieu der ersten Tschechoslowakischen Republik aufzuzeigen sein. Beide Ziele, so partiell und im Grunde zufällig das Belegmaterial auch ist, auf das sie sich im vorliegenden Text stützen, sollen auf dieselbe Pointe hinauslaufen: Für Ludwig Winder sind viel mehr kulturpolitische Kontexte relevant bzw. grundlegend, als die wenigen, in die sein Schreiben bis dato eingebettet wurde, d.h. der jüdische und der der Prager deutschen Literatur (vgl. die Einleitung zum Themenschwerpunkt im vorliegenden Heft u. a. m. ${ }^{1}$ ). Winders Platz unter jüdischen Literaten ist damit gewiss nicht zu bestreiten, genauso wie sein Platz in der Geschichte Prager deutscher Literatur. Aber auch für österreichische Kontextualisierungen bietet Winders Schaffen viele relevante Anknüpfungspunkte (vgl. den Beitrag von M. Weinberg, K. Lahl und W. Müller-Funk im vorliegenden Heft). Der Kontext, dessen Relevanz für Winder im vorliegenden Beitrag skizziert wird, ist derjenige, der sich von der Position eines deutsch schreibenden (aber auch die auf Tschechisch betriebene Kultur, Politik und Literatur berücksichtigenden) Journalisten aus der Tschechoslowakei aus entfaltet.

\section{Deutsche Zeitung Bohemia - ein verlorener Posten?}

Am Sonntag, dem 30. Januar 1927, feierte die Deutsche Zeitung Bohemia ihren hundertsten Geburtstag. Sie tat dies, indem sie in einer Ausgabe erschien, die neun Beilagen hatte und insgesamt 138 Seiten stark war. Neben dem Leitartikel „Die Tradition eines Jahrhunderts“2 vom Chefredakteur des Blattes, Albert Wesselski, prangt auf der Titelseite ein Interview mit dem Staatspräsidenten der Tschechoslowakei, Tomáš G. Masaryk, unter der exklusiven Überschrift „Präsident Masaryk über kulturelle Zeitfragen. Äußerungen für die ,Bohemia““3. (In der rechten Spalte der Titelseite hat noch ein kurzer, telegraphierter, recht phrasenhafter Glückwunsch des österreichischen Bundespräsidenten Michael Hainisch Platz gefunden). Das Interview mit Masaryk hat einen aus tschechoslowakischer Perspektive wahrhaft konstruktiven Grundtenor. Masaryk hebt zunächst - wenig überraschend - die Durchsetzung des „Demokratismus im Ge-

1 Puech, Chantal (2019): Ludwig Winder - das Prosawerk. Wege aus der Unmündigkeit - eine Ethik des Handelns und der Pflicht. Würzburg: Königshausen \& Neumann, S. 35, S. 325 u. a. m.

2 Wesselski, Albert (1927): Die Tradition eines Jahrhunderts. In: DZB 100, Nr. 25, 30. 1., S. 1.

3 F. W. (1927): Präsident Masaryk über kulturelle Zeitfragen. Äußerungen für die „Bohemia“. In: DZB 100, Nr. 25, 30. 1. 1927, S. 1. 
gensatz zum Aristokratismus"4 als das wichtigste kulturpolitische Ereignis der letzten hundert Jahre hervor und bekennt sich zum „Humanitätsideal ${ }^{“ 5}$ des Comenius, das er allerdings „nicht sentimental ${ }^{\text {“6 }}$ aufgefasst wissen will, sondern, wie sich aus dem Weiteren herausstellt, als Handlungsbasis für praktische Politik, die damit demokratisch und humanistisch fundiert wird und somit nationale Motive implizit zweitrangig macht. Der Interviewer der DZB will nämlich auch strittigere Fragen an das Staatsoberhaupt nicht meiden, deren hoher Stellenwert für die Leserschaft der DZB deutlich ist. Eigentlich ließen sich diese Fragen als ,inszenierte Fallen" für den Präsidenten bezeichnen, die indes nicht zuschnappen und die ,aktivistische ${ }^{67}$ Wirkung auf die deutsche Leserschaft der DZB ausstrahlen können. Falle Nummer eins: „Glauben Sie, Herr Präsident, daß die Sendung der tschechischen Nation auch nach den Erfahrungen der neuesten Zeit die Erreichung des Humanitäts-Ideals ist, oder haben Sie sich zu den Anschauungen Palackys [sic], der in der Sendung der tschechischen Nation der Antagonismus gegen das Deutschtum sieht, bekehrt?"8 Masaryk bleibt in der Antwort natürlich bei seinem humanistischen Tschechenbild. Der DZB-Redakteur fragt jedoch noch detaillierter nach: „In der ,Česká otázka' vertraten Sie die Auffassung, daß deutscher und tschechischer Charakter einander sehr ähnelten [...]. Wie erklären Sie, Herr Präsident, den trotzdem bestehenden Antagonismus? "9 Masaryk stützt sich bei der Antwort auf einen Sophismus, dessen deterministische Komponente er offenbar übersieht. Zwischen Nationen, Kirchen oder anderen Menschengruppen, „die einander am nächsten stehen, [bestehen] die schärfsten Antagonismen, "10 meint er. Trotzdem dürften Masaryks Antworten für die Redaktion der DZB zufriedenstellend genug gewesen sein, um sie an die Spitze der Jubiläumsausgabe zu stellen.

Das von Masaryk und der DZB-Redaktion angedeutete Programm ließe sich in historiographischer Begrifflichkeit als ,sudetendeutscher Aktivismus' in der Tschechoslowakischen Republik bezeichnen. Dieser ist auch in der Jubiläumsausgabe prominent vertreten, unter anderem durch den ersten Text der 3. Beilage mit dem Titel „Staaten, Völker und Menschen", dessen Autor der tschechoslowakische Außenminister Edvard (hier Eduard) Beneš ist und der über die vom Völkerbund vorbereitete Abrüstungskonferenz berichtet. In derselben Beilage findet sich auch der umfangreiche, für die Beilage offensichtlich zentrale Beitrag Bruno Kafkas ${ }^{11}$ über die, wie Kafka berichtet,

4 F. W. [Anm. 2], S. 1.

5 F. W. [Anm. 2], S. 1.

6 F. W. [Anm. 2], S. 1.

7 Aktivismus bezeichnete im Diskurs der ersten Tschechoslowakischen Republik die gegenüber der Republik loyale, aktive Einstellung deutschsprachiger politischer Akteure im Staat, allen voran der politischen Parteien, Redaktionen, Vereine usf. Vgl. Broklová, Věra (1999): Politická kultura německých aktivistických stran v Československu 1918-1939. Praha: Karolinum. Broklovás pauschalisierende qualitative Unterscheidungen zwischen der (höheren) „politischen Kultur“ tschechischer und der (niedrigen) „politischen Kultur“ deutscher aktivistischer Parteien sind allerdings von der Hand zu weisen.

8 F. W. [Anm. 2]. S. 1.

9 F. W. [Anm. 2]. S. 1.

10 F. W. [Anm. 2]. S. 1.

11 Bruno Kafka (1881-1931), tschechoslowakischer Abgeordneter für die liberale Deutsche demokratische 
durch Masaryk angeregten Besprechungen über die Teilnahme der Deutschen an der tschechoslowakischen Regierung im Jahre 1924, dem Gründungsakt der jungen Tradition des ,sudetendeutschen Aktivismus“ in der Tschechoslowakei. Auf Seite 3 derselben Beilage steht last but not least der Beitrag des damals (für den „Bund der Landwirte“) amtierenden deutschen Ministers in der tschechoslowakischen Regierung Franz Spina, des wohl bekanntesten Exponenten der ,aktivistischen' Politik der deutschen Parteien in der Tschechoslowakei. Spina zeichnet ein harmonisches Bild Mitteleuropas, dessen Zielgruppe die tschechoslowakischen Deutschen („die politische Brücke nach Deutschland" nennt sie Spina) sind und das im Januar 1927 als durchaus real erscheinen konnte:

\begin{abstract}
„Die tschechoslowakische Republik hat schon aus geographischen Gründen das größte Interesse, zu einem dauernden harmonischen Verhältnis zu dem großen deutschen Nachbarn zu gelangen. Es gibt heute keine stärkere Garantie für die Integrität der Republik als ein Deutschland, das ihr dauernd wohl und freundlich gesinnt bleibt. Was liegt nun näher, um ein solches Verhältnis, wie es ja bereits angebahnt ist, zu vertiefen und zu einem Pfeiler unserer Außenpolitik zu machen? Was liegt nun näher frage ich, als uns Sudetendeutsche zur politischen Brücke nach Deutschland zu benützen? Deutschland hat kein Interesse an der Störung der Sicherheit der Tschechoslowakei als eines seiner wertvollsten und aktivsten wirtschaftlichen Abnehmer; wohl aber hat Deutschland ein Interesse, daß es einem Staat wohl ergehe, wo die stärkste deutsche Minderheit lebt, wohl hat es ein Interesse, daß diese deutsche Minderheit als starkes Element eines benachbarten Staates die Politik dieses Staates in deutschfreundlichem Sinne beeinflusse. Umgekehrt hat die Tschechoslowakei ein noch größeres Interesse, daß diese deutsche Minderheit die Politik Deutschland in einem unserer Republik freundlichen Sinne beeinflusse." ${ }^{12}$
\end{abstract}

Neben win-win-Szenarien, wie Spina eines vorstellt, finden sich in der Jubiläumsausgabe der DZB allerdings auch Beiträge, die die (bis 1938) nicht eingelösten Versprechen der tschechoslowakischen Politiker hinsichtlich der politischen bzw. sprachlichen Selbstbestimmung der ,Sudetendeutschen ${ }^{613}$ einforderten bzw. an sie erinnerten. ${ }^{14}$ Genauso, wie die Jubiläumsausgabe - die ich hier stellverstretend für die gesamte Blattlinie der DZB in der Ersten Republik präsentiere - die verschiedenen Arten des tschechoslowakischen ,Aktivismus' deutschsprachiger Politiker den Appellen an die Einhaltung der Minderheitenpolitik seitens des Staates zur Seite stellte, genauso regional heterogen war, was das kulturelle bzw. literarische Feld betrifft, die Reichweite der Zeitung. Einfacher gesagt

Freiheitspartei, der die DZB am nächsten stand, vgl. Krolop, Kurt (2015): Ludwig Winder. Sein Leben und sein erzählerisches Frühwerk [1967]. Olomouc: UP, S. 52.

12 Spina, Franz (1927): Die Brücke nach Deutschland. In: DZB 100, Nr. 25, 30. 1. 1927, Beilage 3 , S. 3.

13 Der Begriff wurde als in der Tschechoslowakei als neutrale Bezeichnung der gesamten deutschsprachigen Minderheit des Staates benutzt. Erst in den 1920er Jahren setzte er sich allmählich auch als ein Konzept für eine ,eigene' kollektive Identität der deutschsprachigen Bevölkerung in der Tschechoslowakei, einschließlich der Prager Deutschen, durch.

14 z.B. der Beitrag des tschechoslowakischen Senators für die Deutsche Christliche Volkspartei Wilhelm Medinger: Dringlichkeit der Lösung der Sprachenfrage. In: DZB 100, Nr. 25, 30. 1., Beilage 3, S. 3. 
gilt sowohl für die zweite (literarische) Beilage „Dichtung und Bekenntnis“ als auch für die dritte (politische) Beilage „Staaten, Völker und Menschen“, dass sie recht bunte Mischungen von Prag und Provinz, Tschechoslowakei und Sudetendeutschtum, liberal und konservativ darstellen - eingebettet in mehrheitlich deutsche und österreichische Netzwerke. In der Literaturbeilage stehen auf der Titelseite Beiträge von Heinrich Mann, Hans Watzlik und Hedda Sauer Seite an Seite oder auf Seite 4 eine verklärte Naturskizze des böhmischen Regionalklassikers Gustav Leutelt zwischen Texten zweier Grandseigneurs der Prager deutscher Literatur Friedrich Adler und Paul Leppin. Auf die Texte von Edvard Beneš und Bruno Kafka auf Seite 1 der politischen Beilage folgen wiederum neben den der Tschechoslowakei wohlgesinnten Beiträgen von Franz Spina oder Georg Mannheimer auch vielmehr aktivierende als (im tschechoslowakischen Wortgebrauch) ,aktivistische“ Texte wie „Die Sendung der Grenzdeutschen “15 von A. Zycha (Berlin) oder „Vom Staatsbewußtsein der Sudetendeutschen“ von Heinrich Herkner (Berlin). In der von Karl Hans Strobl, der breit von der Entstehungsgeschichte seines Prager Studentenromans Schipkapass berichtet, und Robert Hohlbaum, denen gewiss keine Sympathien zur Tschechoslowakei vorgeworfen werden können, dominierten Beilage „Erinnerung und Erkenntnis" findet sich wiederum ein gezeichnetes Autoporträt samt Widmung an die DZB von Emil Orlik, dem ikonischen Illustrator vieler klassischer Prager deutscher Texte.

All diese Beispiele sollen veranschaulichen, dass die Hypothese vom massiven Bedeutungsverlust der liberalen Bohemia, die bei ihrer konservativ-nationalen sudetendeutschen Zielgruppe bereits seit der Jahrhundertwende nicht mehr ankommen kann, wie sie von Egon Fischer 1934 formuliert und Kurt Krolop wiedergegeben ${ }^{16}$ wird, nicht ganz stimmt. Es wäre auch verwunderlich, es mit einer vierzigjährigen Untergangsphase einer Tageszeitung zu tun zu haben, wie Fischer das nahelegt, in einer Branche, die kaum auf länger bestehende Projekte zurückblicken kann. Die Jubiläumsausgabe von 1927 erweckt jedenfalls nicht den Eindruck eines Niedergangs. Ganz im Gegenteil: Sie kann die prominentesten deutschen, österreichischen, tschechoslowakischen, sudetendeutschen Beiträger aus Politik, Kunst und Wissenschaft gewinnen und kann es sich leisten, verschiedene politische und ästhetische Konzepte zu beherbergen. Zumindest in der 2. Hälfte der 20er Jahre ist die DZB eine Breitband-Zeitung mit primär, sudetendeutscher" Reichweite, im Sinne der umfassenden Bedeutung dieses Wortes, die in der ersten Tschechoslowakischen Republik im Umlauf war.

Die Stellung von Ludwig Winder als Theater- und Kulturredakteur muss unter diesen Umständen auch nicht nur auf eine nur ihm eigene besondere Freiheit ${ }^{17}$ eines angesehenen Redaktionsmitglieds und eines der politischen Generallinie der Zeitung nicht unbedingt folgen müssenden Kulturredakteurs zurückgeführt werden. Winder bewegt

15 „Wahren will ich meine Art und gläubig die Treue halten meinem Volk.“ Zycha, A. (1927): Die Sendung der Grenzdeutschen. In: DZB 100, Nr. 25, 30. 1., Beilage 3, S. 5.

16 Krolop [wie Anm. 11], S. 52: „Der um die Jahrhundertwende einsetzende Niedergang der Bohemia hielt gleichen Schritt mit dem Niedergang des Liberalismus, mit dessen Institutionen und Organisationen das Blatt stets auf das engste verbunden blieb.“

17 Krolop [wie Anm. 11], S. 59-61. 
sich im Hinblick auf weltanschauliche Fragestellungen mit seinen Beiträgen für die DZB in einem recht großen, offenen Aktionsradius, von dessen Prager Mittelpunkt es nahelag, tschechisch- und deutschsprachige Ereignisse und Problematiken zentral, österreichische, deutsche, mitteleuropäische Ereignisse und Problematiken intensiv in den Blick zu ziehen. Aus dieser Position offenbart sich bei der Betrachtung von Winders prosaistischen und journalistischen Beiträgen zu autoritären Figuren eine Einheit, von dieser Position aus lässt sich Winders journalistische Tätigkeit als ,tschechoslowakisch charakterisieren.

\section{Willens- und Machtmenschen des Journalisten Winder}

1967 schrieb Kurt Krolop in seiner Dissertation:

„Ludwig Winder hat während seiner fünfundzwanzigjährigen Tätigkeit als Feuilletonredakteur, Theater- und Literaturkritiker der Bohemia an die 3000 Beiträge für dieses Blatt geschrieben: Theaterkritiken, Buchbesprechungen, Essays, Feuilletons, Glossen und Leitartikel. [...] Eine Untersuchung und Auswertung allein der Theaterkritiken, Buchbesprechungen und kritischen Essays Ludwig Winders ergäbe eine ziemlich lückenlose Geschichte der deutschsprachigen Literatur und der Prager deutschen Theater vom Ausbruch des ersten bis zum Ausbruch des zweiten Weltkriegs. “18

Heute ist die Deutsche Zeitung Bohemia komplett digitalisiert, der OCR-Software der Kramerius-Datenbank verträgt sich allerdings mit der Frakturschrift so schlecht, dass sich auf automatischem Wege nur ein Bruchteil von Winders Beiträgen identifizieren lässt. Wie aufschlussreich und lohnenswert es ist, auf Winders journalistische Texte näher einzugehen, zeigt Peter Becher, ${ }^{19}$ der anhand von diesen Texten die Entwicklung von Winders Einstellung zum ersten Weltkrieg, zum Militarismus allgemein, und nicht zuletzt zur österreichisch-ungarischen Monarchie unter die Lupe nimmt und eine sehr feinfühlige, dynamische und ehrliche Auseinandersetzung des jungen Bohemia-Redakteurs mit diesem Themenkomplex feststellen kann. Ich kann anhand von Winders journalistischen Texten im Folgenden hingegen lediglich den Hinweis untermauern, dass die Beschäftigung mit Willens- und Machtmenschen, die in seinen Romanen mit großer Komplexität bearbeitet wird, auch in seiner journalistischen Tätigkeit einen zentralen Stellenwert einnimmt. Ich beziehe mich dabei auf die DZB-Beiträge Winders, die schon Krolop in einigen seiner legendären Fußnoten anführt. Die Fußnoten gehören größtenteils zum folgenden Abschnitt, in dem Krolop Winders „demokratische Grundhaltung ${ }^{“ 20}$ und seinen wachen Antimilitarismus an Beispielen belegt:

18 Krolop [wie Anm. 11], S. 53.

19 Becher, Peter (2016): Ludwig Winder als Kulturredakteur der Bohemia 1914-1918. In: Höhne, Steffen [et

al.] (hrsg.): Max Brod (1884-1968). Die Erfindung des Prager Kreises. Köln: Böhlau, S. 303-316.

20 Krolop [wie Anm. 16], S. 59. 
„Winder erkannte nicht nur die historische Bedeutung, sondern auch die historische Gerechtigkeit der Oktoberrevolution; er nutzte jede Gelegenheit, gegen den Militarismus aufzutreten; er erhob Anklage gegen die Fememorde des weißen Terrors in Deutschland; während eines Italienaufenthalts im Sommer 1923 lernte er aus eigenen Anschauung den Faschismus kennen, dessen Gefährlichkeit er auch in den spanischen und deutschen Epigonen Mussolinis nicht verkannte.“21

Die Illusionslosigkeit, die Unbestechlichkeit, mit der Winder gegen die autokratisch Herrschenden aller Länder ins Feld zieht, steht etwa seinem Thronfolger-Roman in nichts nach. Es scheint sogar, dass Winders schonungslose Analysen von innerlich leeren Herrscherfiguren eine Art Vorbereitung auf seinen einzigen historisch-politischen Roman (über Franz Ferdinand) sind: Winder scheint psychologisches Material aus dem Inneren der Machtvollen zu sammeln, das in der Regel unzugänglich ist, und sobald er es enttarnen kann, tut er das. Er legt die innere Leere des autoritären Bösen bloß, egal ob es links- oder rechtsautoritär ist oder ob es als stark oder als gütig auftritt. Die Ähnlichkeit zwischen Winders Franz Ferdinand im Thronfolger und seinen Gedanken zum Tagebuch des letzten russischen Zaren, das in deutscher Übersetzung im Jahre 1923 veröffentlicht wurde und von Winder „das grauenhafteste Buch der Weltliteratur“22 genannt wird, ist in der Tat verblüffend. Ganz wie Franz Ferdinand ist Nikolaus zunächst geraume Zeit Thronfolger, er ist ein trockener, pedantischer Geist, ganz wie Franz Ferdinand ist er leidenschaftlicher Jäger („Tag für Tag notiert der Kronprinz die Zahl der erlegten Tie-

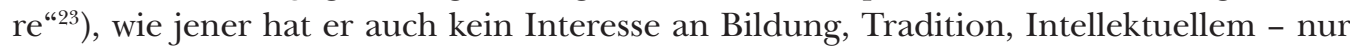
an Macht und Beherrschung („Er lernt nichts, er interessiert sich für nichts, einmal wöchentlich kommt ein Professor, der vorträgt, aber nicht prüfen darf. Der Kronprinz notiert: ,Ich habe eine Stunde dem Professor zugehört.“"24) Nikolaus ist höchstens gelegentlicher sentimentaler Gefühlsausbrüche gegenüber seiner Frau fähig, die er zur Adressatin kitschiger Gedichte macht, genauso wie sie ihn. Winders Entrüstung über das Tagebuch erreicht den Höhepunkt bei Einträgen zu Tagen von Aufständen bzw. Kriegen in Russland, die im Tagebuch meistens überhaupt keine Berücksichtigung finden:

„Im Krieg entpuppt er [Nikolaus] sich. Am 26. Jänner 1904 begann der Krieg. An diesem Tag notiert der Zar: ,Den ganzen Tag waren wir in gehobener Stimmung! Um 8 Uhr fuhren wir ins Theater: es wurde ,Russalka' sehr gut aufgeführt.' am 21. April empfängt er die Nachricht von der großen russischen Niederlage des 19. April. Er notiert: ,Leider wurden außer großen Menschenverlusten auf den Positionen Kanonen und Mitrailleusen gelassen, da alle Pferde getötet waren. Das ist peinlich und schwer!‘ Tags darauf: ,Um 12 Uhr nachts fuhr ich zur Jagd. Diesmal hatte ich Glück und schoß fünf Birkhähne. Die Nacht war wunderschön.““25

21 Krolop [wie Anm. 16], S. 59.

22 Winder, Ludwig (1923a): Das Tagebuch des letzten Zaren. In: DZB 96, Nr. 287, 8. 12., S. 2.

23 Winder [wie Anm. 22], S. 3.

24 Winder [wie Anm. 22], S. 3.

25 Winder [wie Anm. 22], S. 3. 
Winders eigentliches Anliegen mit dem Text ist aber - neben der grundsätzlichen Bildkorrektur des letzten Zaren, der offenbar als „gutmütig, schwach und unglücklich“26 wahrgenommen wurde - die Möglichkeit zu unterbinden, Nikolaus als (rückwärtsgewandte) Projektionsfläche zu benutzen: eine Haltung, die sich genauso gut Winders Thronfolger-Roman als Motto voranstellen ließe: „Dieser Mann hat das Reich Dostojewskis und Tolstois beherrscht. Es ist gut, dass wir ihn endlich ganz kennenlernen. Das Buch wird gewisse Leute vielleicht davon abhalten, die Vergangenheit in rosigem Licht zu sehen. “27

Dass die Gefahr, Europa zu diktatorischen Regimes abgleiten zu sehen, nicht primär von abgesetzten Monarchen, sondern von den neuen „Willensmenschen“"28 in europäischer Politik droht, war Winder klar. Sein Schlüsseltext dazu vom 16. September 1923 heißt "Gefährliches Epigonentum“ und benennt sowohl den Mechanismus der Machtübernahme, als auch die „Psychologie des Falles“29 - sowohl seitens der Diktatoren als auch seitens der Öffentlichkeit, „die innerlich zerrissen, haltlos im Finstern tappend, nach einem starken Mann schreit, der alle Verantwortung auf sich nimmt. " ${ }^{\text {"30 }}$ Auf dieses Bedürfnis der Öffentlichkeit antworten die „Willensmenschen“ durch ein Szenario, das in Italien (Mussolini) und Spanien (de Rivera) gleich sei:

„Ein Mann, der sich des Vertrauens der Truppen bemächtigt, läßt sie einfach losmarchieren, besetzt die politische wichtigsten Städte des Landes, telegraphiert dem König, die bisherige Regierung müsse abgesetzt werden und bildet, im ,freundschaftlichen Einvernehmen“ mit dem Regenten, eine neue Regierung. [...] Die Könige werden nicht mehr gestürzt, man läßt sie in ihren Schlössern wie bisher das bedeutungslose Zeremoniell erfüllen - für so ungefährlich werden sie von den militärischen Diktatoren gehalten. Das Selbstbewusstsein der Soldatenführer ist ins Grenzenlose gewachsen.“31

An der Machtübernahme beteiligen sich also maßgeblich die Adeligen, die den Schein des Status quo wahren, indem sie das Spiel der neuen Machthaber sanktionieren, aber völlig ohne Einfluss auf die politische Praxis sind. Nach diesen Noten erfolgt auch die Übergabe der absoluten Macht über die (fiktive) böhmische Stadt Boran an den ,Willens- und Machtmenschen' Adam Dupic in Winders Roman Die nachgeholten Freuden (1927), in dem die Zeitenwende von 1918 eben durch die Abdankung des Adels auf dieselbe Art und Weise wie im „Gefährlichen Epigonentum“ gekennzeichnet wird.

Winder betrachtet das Problem prinzipiell als den Gegensatz zwischen Diktatoren auf der einen und der Demokratie auf der anderen Seite, und neben der Einsicht, dass Mussolini und Rivera auf den „Schrei nach dem starken Mann“ antworten, lautet sein

26 Winder [wie Anm. 22], S. 2.

27 Winder [wie Anm. 22], S. 3.

28 Winder, Ludwig (1926a): Erbliche Diktatur. In: DZB 99, Nr. 152, 27. 6., S. 4. In diesem Text Winders wird Mussolini „Willensmensch“ genannt.

29 Winder, Ludwig (1923b): Gefährliches Epigonentum. In: DZB 96, Nr. 216, 16. 9., S. 1.

30 Winder [wie Anm. 29], S. 1.

31 Winder [wie Anm. 29], S. 1-2. 
Hauptkritikpunkt, dass die beiden eben „Epigonen“ sind und außer Willensstärke nur plagiierte politische Rezepte parat haben: „Auch Mussolini ist Epigone; es wäre töricht, daran zu zweifeln, daß sein Vorbild Lenin ist. Ob Linksdiktator oder Rechtsdiktator - sie

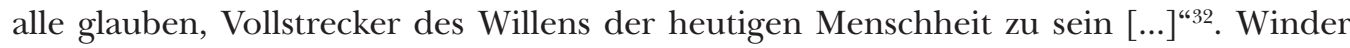
unterschätzt diese Gefahr für die Demokratie nicht:

„Es ist unmöglich, daß diese Ereignisse und Erscheinungen den westlichen Völkern, die sich Stätten der Demokratie dünken, kein Herzklopfen verursachen. [...] Die Entscheidung für das westliche Europa heißt: Deutschland. Wenn der Westen nicht Frieden mit Deutschland macht, wird Europa in absehbarer Zeit von einigen militaristischen Diktatoren beherrscht sein. Das würde das Ende Europas bedeuten “33,

schreibt Ludwig Winder im Jahre 1923.

Kaum zwei Monate später, am 9. November 1923, ereignete sich in München der Hitler-Ludendorff-Putsch, der in der DZB u.a. in einem anonymen Kommentar besprochen wurde, dessen Autor mit großer Wahrscheinlichkeit Ludwig Winder war. ${ }^{34}$ Auch in diesem Text wird das günstige öffentliche Bild des autoritären Politikers in Zweifel gezogen, es werden - genauso wie bei Mussolini und de Rivera - Hitlers Willensstärke und sein Selbstbewusstsein thematisiert, seine autokratische Ambition wird, trotz seiner 1923 noch „bescheidenen“"35 Rhetorik, dargelegt. Beim ersten Blick könnte es erscheinen, dass selbst der hellhörige Winder Hitler im Lichte des schnell missglückten Putsches unterschätzt hat, er nennt ihn doch ironisch-herablassend den deutschen „Eintags-Mussolini“ und spricht ihm schließlich auch diese fragwürdige Auszeichnung ab: „Indessen hatte sich erwiesen, daß er nicht einmal der Vergleich mit Mussolini ausgehalten hat. Der deutsche Mussolini ist Hitler nicht. “36 Winder ist aber klar, dass die Tendenz zu bewaffneten Machtübernahmen in europäischen Staaten aktuell stark ist, und dass sie auch an Deutschland nicht vorbeigehen wird, auch wenn der Hitlerputsch gescheitert ist: „Der deutsche Mussolini ist Hitler nicht. Er war es vorgestern [d.h. am 8. 11. 1923, JB]. Wer wird es übermorgen sein?"37

Aus der Charakteristik der Deutschen Zeitung Bohemia, wie sie sich aus der Analyse der Jubiläumsausgabe im Januar 1927 ergibt - nämlich als eine liberale Zeitung in dem Sinne, dass sie in literarisch-kulturellen, aber auch politischen Belangen eine Vielfalt von Perspektiven beherbergen und verschiedene Zielgruppen bedienen konnte - und aus Winders konsequenter und mit seinem Romanwerk aufs Engste verwobener journalistischer Kritik am zeitgenössischen Autoritarismus und Militarismus zeichnet sich ein schlüssiges Bild ab. Zum einen führt Winder genau die Prinzipien der Demokratie und

32 Winder [wie Anm. 29], S. 1.

33 Winder [wie Anm. 29], S. 2.

34 Krolop ([wie Anm. 11], S. 59 und 245) schreibt den Text Winder zu, und in der Tat liest er sich wie die nächste Folge von Winders Analyse der Machtübernahme durch de Rivera in Spanien.

35 Winder, Ludwig (1923c): Der deutsche Eintags-Mussolini. In: DZB 96, Nr. 263, 10. 11., S. 3.

36 Winder [wie Anm. 35], S. 3.

37 Winder [wie Anm. 35], S. 3. 
des Humanismus im Schilde, durch die sich auch - repräsentiert durch das MasarykInterview an der prominentesten Stelle der Jubiläumsausgabe - seine Redaktion präsentierte. Diese Prinzipien setzt er in seiner Kritik an historischen (Zar Nikolaus) und zeitgenössischen (Mussolini, de Rivera, Hitler), Willens- und Machtmenschen' konsequent um. Zum anderen lässt sich beobachten, dass Winder seine Perspektive zunächst im journalistischen Werk, anhand von konkreten politischen Akteuren formulierte. Erst nachher, etwa mit den Nachgeholten Freuden oder dem Thronfolger, was seine geschichtspolitisch am deutlichsten zu verortenden Romane ${ }^{38}$ sind, hat er als Romancier explizit politische Prosa gewagt. Winders Essayistik (in der DZB), die zum großen Teil im tschechoslowakischen Kontext aufgeht, lässt sich somit zumindest als eine Art Labor angehen, aus dem schließlich größere Prosa-Legierungen hervorgegangen sind.

\section{Winders tschechoslowakische Bezugnahmen}

Winder berichtet in der DZB von Hunderten von kulturellen bzw. politischen Ereignissen, die sich in der Tschechoslowakei abspielten, viele rein tschechischsprachige miteingerechnet. Aus Winders Besprechung ${ }^{39}$ der Premiere der die Jahrhundertwende „liebevoll-träumerisch bespöttelnden“ Komödie Der Strohhut (tsch. Slaměný klobouk) des Befreiten Theaters (tsch. Osvobozené divadlo) von Jiřri Voskovec und Jan Werich beispielsweise geht deutlich hervor, dass der Rezensent das Stück zwar - wie auch die übrigen des Autorenduos - genossen hat, dass er aber von diesem Ensemble aktuellere Stoffe und stärkeres politisches Engagement erwartet. „Voskovec und Werich“, schreibt Winder, „die unbekümmert genialen Bänkelsänger und Zeitkritiker, zeigen nicht ihre scharfen Zähne. ${ }^{* 40}$ Häufiger als gattungsmäßig ,reine“ Besprechungen von Büchern oder Theateraufführungen entfalten sich Winders ,tschechoslowakische‘ Texte zu komplexeren, oft auch international angelegten Auseinandersetzungen mit breiterer Problematik, z.B. der der späten, verheerenden Folgen des Weltkriegs. Im Leitartikel „Mörder und Gemordete“ ${ }^{“ 41}$ vom 24. November 1922 geht der von der Statistik der Mährisch-Ostrauer Polizeidirektion aus, laut der in der Zeit vom 1. Februar bis zum 15. November 1922162 Morde verübt wurden, darunter 108 Raubmorde. Dies führt Winder auf den Weltkrieg, aber auch auf die soziale Krisensituation zurück:

„Ein Menschenleben ist während des Krieges wertlos geworden. Tausende haben die Verlustlisten wie einen uninteressanten Geschäftsbericht überflogen, von Tag zu Tag stumpfer, kaltblütiger, teilnahmsloser. Als der Krieg aus war, begann der Valutakrieg, der den früheren vergessen machte. Die nächsten Folgen: Industriekrise, ein ungeahntes Anwachsen der Arbeitslosigkeit.

38 Sieht man von den im Exil entstandenen Zeitromanen Die Novemberwolke und Die Pflicht ab, die auch sehr konkret mit einer bestimmten historischen Situation arbeiten.

39 Winder, Ludwig (1934): Das neue Stück des „Befreiten Theaters“. In: DZB 107, Nr. 50, 1. 3., S. 5.

40 Winder [wie Anm. 39], S. 5.

41 Winder, Ludwig (1922): Mörder und Gemordete. In: DZB 95, Nr. 276, 24. 11., S. 1. 
Im Ostrauer Industriegebiet lebt ein Heer von Arbeitslosen: dort sind die meisten Verbrecher zu hause. “42

Die unerfreuliche Statistik wird von Winder allerdings im Weiteren als Sprungbrett zur Kritik an der (1919 bis 1922 vorwiegend sozialistischen) tschechoslowakischen Regierung benutzt, indem die Erfolge der internationalen Pazifisten gegen sie ausgespielt werden. „An diesen Erfolgen“, behauptet Winder,

„hat die Tschechoslowakei [...] keinen Anteil. In der Tschechoslowakei wimmelt es von Uniformen, sichtbaren und unsichtbaren. Ein frischfröhlicher Krieg würde so manchem in den Kram passen, der noch vor wenigen Jahren aus Nationalismus Pazifist zu sein vorgab. Diese mögen bedenken, daß im Krieg wie im Frieden weder Deutscher noch Tscheche, weder Christ noch Jude vor dem unbekannten Mörder sicher ist. Die menschliche Bestialität kennt weder Nationalität noch Rasse. “43

Geht hier Winder auf Distanz zur tschechoslowakischen Politik, ist das keine nationale Distanz eines Deutschen bzw. eines deutsch sprechenden Juden zum ,tschechoslowakischen' Staat, es ist auch keine Distanz etwa eines Konservativen gegenüber sozialistischen Regierungen. Es ist die Distanz eines antimilitärisch gesinnten und humanistisch denkenden Menschen zu einem Staat, der den Zusammenhang zwischen psychischer Belastung und sozialer Not auf der einen und der Gewalt auf der anderen Seite nicht zu sehen scheint und der damit aus gesamteuropäischer Perspektive hinter andere Staaten zurückfällt.

Auch Winders Artikel zum kulturellen Leben Prags bzw. der Tschechoslowakei legen diese Art von konstruktiver, als Erziehung gedachter Kritik an den Tag. Auch hier ist sie frei von ideologischen, geschweige denn nationalen Dimensionen. Bleiben wir beim Thema des Antimilitarismus, lässt sich das Beispiel von Winders Besprechung der Prager Aufführung des Schauspiels Das Grabmal des unbekannten Soldaten von Paul Raynal heranziehen. Das Stück stellt für Winder den „wahren Pazifismus“ dar, wie er „aussehen müßte - wenn die Menschheit nach dem Krieg alle Konsequenzen gezogen hätte." ${ }^{44}$ Der „wahre Pazifismus“ des Stücks beschäme laut Winder „alle pazifistischen Schönredner“45, da es aufs Eindringlichste zeige, dass „kein Glück, auch das reinste nicht, [...] der mörderischen Gewalt des Krieges standhalten “46 kann. Diese unangenehme, tragische Wahrheit, die weder den „Patrioten“ noch den „pazifistischen Schönrednern“ passe, werde von der Nachwelt immer vergessen - auch darin ist sich Winder mit der Aussage von Raynals Stück einig, und macht es „Prag - und [...] der ganzen Welt" ${ }^{47}$ zum Vorwurf.

42 Winder [wie Anm. 41], S. 1.

43 Winder [Anm. 41], S. 1.

44 Winder, Ludwig (1926b): Das Grabmal des unbekannten Soldaten. In: DZB 99, Nr. 143, 17. 6., S. 6.

45 Winder [wie Anm. 44], S. 6.

46 Winder [wie Anm. 44], S. 6.

47 Winder [wie Anm. 44], S. 6. „Während an der Kasse des Neuen Theaters, wie in der Pause berichtet wurde, um Eintrittskarten zu der Operette ,Uschi` förmlich gekämpft wurde, mußte Reynals Dichtung vor 
Großen symbolischen Wert hat die bekannte Tatsache, dass Winders letzter Beitrag für die DZB der Nachruf auf Karel Čapek ${ }^{48}$ war. Im Zusammenhang des vorliegenden Aufsatzes ist es aber noch wichtiger, dass Winder den Text nicht nur als Nachruf auf einen Dichter einrahmt, sondern als Nachruf auf die erste Tschechoslowakische Republik, die er Čapek förmlich gleichsetzt. Čapek „ging [dahin], als die Ideale seines Lebens dahingegangen waren“49, und er wird von Winder klipp und klar „Demokrat“ genannt, auf seine Nähe zu Masaryk und - mit kleinem Vorbehalt - zu Beneš wird explizit hingewiesen. ${ }^{50}$ Diese demokratische ,Grundkonstante' der Tschechoslowakei, die mit Čapek „dahingegangen“ ist, ist auch für Winder von zentraler Bedeutung. Er vergleicht diesen Verlust mit dem anderen Verlust, den die Tschechoslowakei nicht ganze drei Monate zuvor erlitten hat: nämlich das Abtreten der mehrheitlich deutschsprachigen Grenzregionen Böhmens und Mährens an NS-Deutschland nach dem sog. Münchner Abkommen. Für Winder ist der Verlust der ,Čapek-Dimension“ der Tschechoslowakei „kaum weniger schmerzlich als der Verlust einer Provinz “51. Dies macht die Profundität von Winders Sichidentifizieren mit der tschechoslowakischen Demokratie erkennbar, der er auf diesem Wege nachtrauert, auch wenn nahezu keine deutschsprachige Bevölkerung mehr im territorial beschnittenen Staat lebt. Auf der anderen Seite in an Winders Čapek-Nachruf auffällig, dass Winder trotz enormer Sympathien weder den toten Dichter noch ,seinen“ Staat - wenn auch implizit - ,unser“ nennt. In Nachruf heißt es immer wieder: „Die Tschechen haben den Schriftsteller verloren, der von allen zeitgenössischen Künstlern und Schriftstellern die größte Geltung in der Welt hatte. “52 Oder: „[...] Čapek repräsentierte eine Provinz europäischen Geisteslebens, die der tschechischen Nation Ruhm und Ehre brachte und seinem Volke Freunde schuf wie kaum ein Politiker."53 Oder: „Wer aber etwas von Čapek kennengelernt hatte, war gut auf die Tschechen zu sprechen." ${ }^{54}$ Das sprachlich-nationale Unterscheidungskriterium, das ihn und seine Leser von Čapek trennt, wird von Winder völlig automatisch eingesetzt, was zu dieser Zeit wohl auch nicht anders denkbar war. Im Lichte dieser prinzipiellen, allem Anschein nach nicht hinterfragten Distanz zu den Tschechen als einer sprachlich-kulturellen Formation als dem ,Anderen“ im gemeinsamen Staat erscheint die konstruktive tschechoslowakische Fundierung Winders, die im vorliegenden Text lediglich skizziert werden konnte, noch wertvoller.

\footnotetext{
leerem Hause gespielt werden. Es war beschämend. In dem Stück wird gesagt, das Entsetzlichste im Krieg sei die Gewißheit, daß die Menschheit alle Opfer und alle Geopferten bald vergessen werde. Gestern sah man, wie wahr dieser Satz ist. Man vergißt nur zu gern, man will an den Krieg nicht erinnert werden, man geht lieber zu ,Uschi““.

48 Winder, Ludwig (1938): Karl Čapek †. In: DZB 111, Nr. 305, 28. 12., S. 1.

49 Winder [wie Anm. 48], S. 1.

50 Winder [wie Anm. 48], S. 1. „Er war der Schüler und Freund Masaryks, er galt auch als Freund Benešs.“

51 Winder [wie Anm. 48], S. 1.

52 Winder [wie Anm. 48], S. 1.

53 Winder [wie Anm. 48], S. 1.

54 Winder [wie Anm. 48], S. 1.
} 


\section{Quellen und Literatur}

Becher, Peter (2016): Ludwig Winder als Kulturredakteur der Bohemia 1914-1918. In: Höhne, Steffen [et al.] (hrsg.): Max Brod (1884-1968). Die Erfindung des Prager Kreises. Köln: Böhlau, S. 303-316.

Broklová, Věra (1999): Politická kultura německých aktivistických stran v Československu 19181939. Praha: Karolinum.

F. W. (1927): Präsident Masaryk über kulturelle Zeitfragen. Äußerungen für die „Bohemia“. In: DZB 100, Nr. 25, 30. 1. 1927, S. 1.

Krolop, Kurt (2015): Ludwig Winder. Sein Leben und sein erzählerisches Frühwerk [1967]. Olomouc: UP.

Medinger, Wilhelm: Dringlichkeit der Lösung der Sprachenfrage. In: DZB 100, Nr. 25, 30. 1., Beilage 3, S. 3.

Puech, Chantal (2019): Ludwig Winder - das Prosawerk. Wege aus der Unmündigkeit - eine Ethik des Handelns und der Pflicht. Würzburg: Königshausen \& Neumann.

Spina, Franz (1927): Die Brücke nach Deutschland. In: DZB 100, Nr. 25, 30. 1. 1927, Beilage 3, S. 3. Wesselski, Albert (1927): Die Tradition eines Jahrhunderts. In: DZB 100, Nr. 25, 30. 1., S. 1.

Winder, Ludwig (1922): Mörder und Gemordete. In: DZB 95, Nr. 276, 24. 11., S. 1.

Winder, Ludwig (1923a): Das Tagebuch des letzten Zaren. In: DZB 96, Nr. 287, 8. 12., S. 2-3.

Winder, Ludwig (1923b): Gefährliches Epigonentum. In: DZB 96, Nr. 216, 16. 9., S. 1-2.

Winder, Ludwig (1923c): Der deutsche Eintags-Mussolini. In: DZB 96, Nr. 263, 10. 11., S. 3.

Winder, Ludwig (1926a): Erbliche Diktatur. In: DZB 99, Nr. 152, 27. 6., S. 4.

Winder, Ludwig (1926b): Das Grabmal des unbekannten Soldaten. In: DZB 99, Nr. 143, 17. 6., S. 6. Winder, Ludwig (1934): Das neue Stück des „Befreiten Theaters“. In: DZB 107, Nr. 50, 1. 3., S. 5. Winder, Ludwig (1938): Karl Čapek †. In: DZB 111, Nr. 305, 28. 12., S. 1.

Zycha, A. (1927): Die Sendung der Grenzdeutschen. In: DZB 100, Nr. 25, 30. 1., Beilage 3, S. 5.

Mgr. Jan Budňák, Ph.D. / budnak@phil.muni.cz

Masarykova univerzita, Filozofická fakulta, Ústav germanistiky, nordistiky a nederlandistiky Arna Nováka 1, 60200 Brno, CZ 
